\title{
Cognitive-Behavioural Therapy for First Episodes of Psychosis: A Case Report on the Application of a Multicomponent Treatment Protocol in Italy
}

\author{
Sandro Domenichetti ${ }^{1}$, Andrea Pozza ${ }^{1,2,3, *}$ \\ ${ }^{1}$ Department of Mental Health of Florence Healthcare Services, Via Fanfani, Florence, Italy \\ ${ }^{2}$ Department of Experimental and Clinical Medicine, University of Florence, Largo Brambilla, Florence, Italy \\ ${ }^{3}$ Miller Institute of Behavioural and Cognitive Psychotherapy, Corso Torino, Genoa, Italy \\ *Corresponding author: andrea.pozza@unifi.it, apsycho@hotmail.it
}

Received August 12, 2014; Revised October 14, 2014; Accepted October 24, 2014

\begin{abstract}
Objectives: Although pharmacotherapy with antipsychotic medication is an effective intervention for early psychosis (EP), drop out from treatment and relapse rates appear still relatively high. In the last decade, there is growing interest in Cognitive-Behavioural Therapy (CBT) as early intervention for EP. Some studies suggest that CBT may be an effective adjuvant to pharmacotherapy to reduce risk of development of chronic psychosis in individuals who experienced first episodes. Symptoms of depression and social anxiety are very common among patients experiencing first episode psychosis, thus impairing quality of life and functioning. This aspect may create a negative vicious cycle, that may reinforce feelings of learned helplessness and hopelessness with regard to psychosis diagnosis, thus increasing vulnerability to relapse in further episodes. However, there is little knowledge on the use of CBT to target both reoccurrence of psychotic episodes and symptoms of depression and social anxiety secondary to first psychotic episodes. The current case report presents the administration of a modular CBT semi-manualized intervention to a young patient to enhance prevention of psychosis and target symptoms of social anxiety and depression following first psychotic episodes. Methods: Treatment consisted in 21 individual weekly sessions, delivered in an outpatient setting, involving two phases. The first one targeted prevention of psychotic episodes. Case formulation was developed according to cognitive behavioural model of psychosis. Psychoeducation on early signs and vulnerability factors of relapse in psychotic episodes was delivered in order to promote patient's selfmonitoring and insight on positive symptoms prodromes. The second treatment phase targeted symptoms of depression and social anxiety. Homework based on behavioural activation were assigned in order to address depressive symptoms, and cognitive restructuring was used to normalize dysfunctional beliefs. A social skills and assertiveness training was used to target social anxiety. CBT treatment was delivered in combination with medication. Clinical interviews were conducted at pre-, immediate post-treatment, and at 5-month-follow-up, to evaluate reoccurrence of psychotic episodes, and changes in depression, social anxiety, and functioning. Results: As observed by clinical interviews, the patient did not experience further relapse in psychotic episodes. In addition, it was observed a reduction in depressive symptoms and social anxiety through the increase of the number of daily activities, social relations, work and academic engagement. Conclusions: Important limitations of the study are discussed. Future research using large randomized controlled trials with longer follow-up assessments should evaluate the effectiveness of CBT to target depressive symptoms and social anxiety for patients with EP.
\end{abstract}

Keywords: cognitive behavioural therapy, early psychosis, early intervention, case report

Cite This Article: Sandro Domenichetti, and Andrea Pozza, "Cognitive-Behavioural Therapy for First Episodes of Psychosis: A Case Report on the Application of a Multicomponent Treatment Protocol in Italy." Research in Psychology and Behavioral Sciences, vol. 2, no. 3 (2014): 66-74. doi: 10.12691/rpbs-2-3-3.

\section{Introduction}

\subsection{Early Intervention in Psychosis}

There is growing interest by researchers and practitioners on early intervention in psychosis [1,2]. Some studies suggested that longer the patients do not receive treatment for early psychosis (EP), lower is the probability of symptom remission, thus increasing risk for the development of a chronic psychotic disorder [2,3]. Moreover, early intervention may reduce healthcare costs associated to further hospitalizations and supportive treatments for informal caregivers of the patients [4].

A number of trials have demonstrated that pharmacological treatments with antipsychotic drugs are an effective strategy for EP [5,6]. However, such an efficacy seems to be complicated by some critical factors. During first years of treatment, approximately $60 \%$ of 
patients tend to show poor adherence with antipsychotics; of those patients about $40 \%$ early discontinue medication within six months, and over 50\% show only intermittent adherence with pharmacotherapy [7]. Moreover, after first episode relapse seems to be relatively common within one year [7]. Furthermore, recovery on functioning (on social, professional, and interpersonal areas) may not be a consequence of remission in positive symptoms [7], and the persistence of disability may increase risk for relapse [7].

\subsection{Cognitive Behavioural Therapy for Early and First Episodes of Psychosis}

Recently, multicomponent programs have been developed for individuals experiencing first psychotic episodes, incorporating medication and psychotherapy components, such as assertive outreach and psychosocial treatments $[8,9]$.

Such programs include cognitive behavioural therapy (CBT) in combination with antipsychotic medications [10]. CBT approaches for EP typically focus on individualized case formulation, psychoeducation and normalization of anomalous experiences, relapse prevention of further episodes, and treatment of secondary psychological problems, such as depression and anxiety [10]. Early intervention with CBT also aims to increase adherence to medications, minimize the negative effects related to first psychotic symptoms and hospitalizations, and, finally, improve global functioning in order to enhance remission [10].

A relevant proportion of young patients tends to achieve remission of positive symptoms within one year after psychotic episodes [7]. Comorbid depression and social anxiety are relatively frequent clinical conditions following first episodes of psychosis. With a prevalence rate of approximately $50 \%$ of cases, post-psychotic depression is a significant problem in EP, which can slow recovery, negatively affect quality of life, and predict hopelessness and suicidal thinking [11,12,13]. Social anxiety is frequently reported as an impairing condition during first years following first-episode psychosis as well, with a prevalence rate ranging between 43 to $50 \%$ [14].

Even though the growing interest on EP, to date the majority of research has been conducted on patients with a long-established diagnosis of psychotic disorders [eg, 15], and only a limited number of contributions has been published on CBT for EP. In addition, evidence to date still appears inconsistent. In a meta-analysis conducted on seven randomized controlled trials on EP, Hutton and Taylor [1] reported that CBT produced a 50\% reduction in the relative risk of developing psychosis compared to treatment as usual or control conditions at 18-24 or longer follow-up assessments. However, CBT did not produce significant gains than control conditions on quality of life and functioning outcomes.

Alvarez-Jimenez [16] conducted a recent meta-analysis on psychosocial treatments for patients with first-episode psychosis to prevent second episodes. Findings from three randomized controlled trials suggested that CBT was not superior to supportive counseling and treatment as usual. Moreover, the addition of CBT to a specialist program for EP compared to a specialist program for EP alone, did not show significant advantages in favor of CBT [16].
Persistence of depressive and social anxiety symptoms may moderate progression towards a chronic psychosis in individuals experiencing first episodes. As suggested by some authors, CBT treatments addressing specific symptoms may be more effective than treatments focusing exclusively on general psychotic symptoms $[17,18,19,20]$. Further contributions on the use of evidence-based CBT techniques with a modular approach for EP are required with the aim to identify tailored treatments targeting potential vulnerability factors for relapse and long-term disability, such as depression and social anxiety [eg, 21].

\subsection{Early Intervention on Psychosis in Italy}

From 1999 an early intervention program, the PROGRAMMA 2000, has been developed for individuals experiencing first episodes of psychosis in the Italian healthcare context [22]. The program consisted of a multicomponent treatment, including medication, psychoeducation, cognitive restructuring, problem-solving and assertiveness training. In a recent study on a sample of 132 individuals at first episode of psychosis, Cocchi et al. [22] reported a significant improvement on global functioning at 6- and 12-month follow up.

More recently, in a non-randomized comparative trial on 46 individuals with first-episode psychosis, Cocchi et al. [4] compared the effectiveness of the PROGRAMA 2000 interventions with a routine intervention (RI), consisting of pharmacotherapy and supportive psychotherapy without therapeutic components specifically targeting prevention of psychosis, such as CBT techniques. Results showed that patients on the PROGRAMMA 2000 arm had lower scores relative to those on the other arm on the Health of Nation Outcome Scales at 5-year follow-up. However, differences between the two treatment conditions did not result significant, and patients on the PROGRAMMA 2000 did not achieve reliable change to a greater extent compared to those on the RI [4]. In addition, the PROGRAMMA 2000 seemed to be associated with greater reductions in healthcare costs relative to RI in terms of new hospitalizations or day-hospital visits, although this difference did not reach the significance level [4].

\subsection{Rationale for the Current Study}

Although the promising evidence, from our review of the literature there is still a limited number of contributions on the application of CBT to target both prevention of positive symptoms and post-psychotic symptoms, such as social anxiety, depression, and lower functioning. In addition, findings reported to date appear inconsistent about the effectiveness of CBT for postpsychotic symptom outcomes. This aspect suggests the need for tailored interventions addressing also secondary problems associated to EP.

Persistence of depressive symptoms and social anxiety seems to be relatively common among patients who experience phases subsequent to first psychotic episodes $[11,12,13,14]$. This aspect may significantly interfere with quality of life of the patients and increase their vulnerability to relapses $[11,12,13,14]$.

Therapeutic goals for this type of patients may be significantly different from those goals set for patients with a chronic psychosis [17]. As recently suggested by 
some authors [eg, 17-20], relapse prevention focusing exclusively on antecedents of earlier psychotic episodes (through psichoeducation, self-monitoring of prodromals, and cognitive restructuring of dysfunctional beliefs hypothesized to be a vulnerability factor for acute episodes) might be insufficient. It has been proposed that relapses involve also processes quite different from those related to the development of positive symptoms $[23,24,25]$. Thus, to be effective, CBT should focus also on functioning of the patients during the post-psychotic phase.

\subsection{Objectives}

The current case report aims to describe the application of a CBT intervention to the clinical case of a young patient, who experienced phases subsequent to first brief psychotic episodes. The intervention has been conducted as adjuvant treatment to pharmacotherapy with antipsychotic drugs. The intervention consisted of components targeting relapse prevention of further psychotic episodes and addressing post-psychotic anxiety, depression, and functioning. In the current case report the use of CBT components to this clinical case, their rationale and application with regard to the phases of treatment course are described.

\section{The Case of $M$.}

M. is a 28-year old patient, living in Florence. From 2004 he attends the Centre for Mental Health of Florence, a community outpatient mental health service. In July 2013 he is assigned to a CBT treatment program offered at our service, after having been hospitalized at the psychiatry ward of the University hospital of Florence for a brief psychotic acute episode. Before undergoing CBT, M. had not been on psychotherapy. During CBT treatment course, M. received Aripiprazole, which was subsequently replaced with Olanzapine due to its side effects.

M. accessed our service through his mother, who convinced him to seek help in January 2004 after a hospitalization at the Emergency Department of the Careggi University Hospital of Florence. During the earlier 20 days, he had presented delusional ideation with megalomaniacal and mystic aspects, cenestopathic hallucinations, and ritual-like stereotypical movements. During the last month he had lost $8 \mathrm{Kg}$. When he accessed our service, he was prescribed Aloperidol $6 \mathrm{mg} / \mathrm{die}$, Valproate sodium $400 \mathrm{mg} / \mathrm{die}$, and Lorazepam 2,5 mg/die. His mother told that from several days she was worried about the rapid worsement of his son on school performances. Until that period he appeared a normal student.

At the first interview with the psychiatrist the patient appeared very shy and scared. After the patient had accessed the service, pharmacotherapy was modified prescribing esclusively Risperidone $4 \mathrm{mg} / \mathrm{die}$. In the subsequent weeks recovery in functioning and academic performance was observed. However, M. began to present apathy and tremors, which were interpreted as side effects of Risperidone. Thus, Risperidone was replaced with Olanzapine $10 \mathrm{mg} /$ die. During subsequent days an improvement was observed but apathy, abulia, difficulties keeping concentration, and low self-esteem indeed persisted. Thus, Fluoxetine $20 \mathrm{mg} /$ die was prescribed.

M. applied to an undergraduate course for healthcare professionals and passed the entry exam. In 2007 when he was about to graduate, another psychotic episode was observed with clinical characteristics very similar to the earlier ones: he had stopped medication. Pharmacotherapy with Risperidone was reintroduced, then a strong improvement was observed again, but residual symptoms of asthenia, abulia, and apathy did persist.

M. graduated, then began a professional internship, which was not renewed due to the slowness of the patient. During these years, after the end of two intimate relationships with two girls older than him, M. had two severe psychotic episodes characterized by bouffées delirantes states.

Since the beginning of $2013 \mathrm{M}$. has been a consumer of psychoactive drugs. During the CBT treatment course the patient did not consume psychoactive drugs since he had stopped about one month before. M. has a familiarity for psychosis as his paternal grandmother has a diagnosis of schizoprenia. In addition, his father suffers from obsessive-compulsive disorder.

M. tells a story about himself: "When I was young I had uncontrollable reactions. Specifically, I was an aggressive boy who often got angry. At high school I behaved as a bully with shy peers imitating the most tough of my mates. However, I did not talk with my parents about my behaviours and perceptions at school. At home I was not used to talk with my parents about my feelings, needs and interests, as I acritically accepted anything they wanted for me.

When I was 13-year old at the camp, I had a girlfriend. Although I was affectionate to her, I never went to meet her and never demonstrated my love to her.

When I was 14-year old, I began smoking sigarettes, drinking, and withdrawing into myself. During high school I began to use psychoactive substances (cannabis, ecstasy, cocaine). During that period obsessive illogical thoughts about my body began to come to my head, then I began to do odd things as trying to struggle with gravity or to switch on the engine of my moped without the key. During that period I usually went out with my friends but I was passive, and I was not used to propose ideas. I did not socialize with peers at university, and I was not interested in topics of my courses. Although I continuosly used cannabis, cocaine, and alcohol, I could graduate at university.

Subsequently, I did an internship, but I still felt like spaced out, because when physicians were absent at work I masturbated. Then, I did some open competitions around Italy to find a job, but when I was at home I still used psychoactive substances.

Next, I did voluntary service at an outpatient centre for dependences, but I still continued abusing substances. Finally, in my last job I very much enjoyed working, but I continued abusing substances due to my silliness, then I was hospitalized again.

My family was very helpful for me since my parents always supported me, and without their support I would not be here to write this story about myself”.

\section{Method}




\subsection{Assessment of Psychotic Symptoms, Anxiety, and Depression}

Two independent diagnosticians (a psychiatry and a clinical psychologist) conducted diagnostic interviews at pre-treatment, and, specifically through semi-structured interviews they independently conducted the assessment on psychotic symptoms, social anxiety, and depression at pre-, post-treatment, and at a 5-month follow-up.

\subsection{CBT Intervention}

Overall, the CBT intervention consisted of 21 one-hour weekly individual sessions, which were based on a semimanualized approach inspired to a CBT protocol for early psychosis [17]. Sessions were conducted tailoring the intervention on the patient's priorities. A modular approach was used with the aim to target both psychotic episodes relapse prevention and depression and social anxiety. Sessions were conducted by a cognitivebehavioural clinical psychologist. CBT sessions were monitored since therapist was supervised by another therapist. Therapeutic components of the CBT intervention are provided in Table $\mathrm{I}$ as a function of treatment phases.

Table 1. Overview of the components of the CBT intervention for the case of $\mathrm{M}$

\begin{tabular}{ccc}
\hline Protocol phases & Sessions & Components \\
\hline Phase I: & $1-3$ & List of problems and working alliance \\
\cline { 2 - 3 } \begin{tabular}{c} 
Assessment \\
Phase II: Relapse \\
prevention and \\
\cline { 2 - 3 } $\begin{array}{c}\text { intervention on } \\
\text { depression }\end{array}$
\end{tabular} & $4-5$ & Case formulation \\
\cline { 2 - 3 } and social anxiety & $9-21$ & Psychoeducation on psychotic episodes \\
\cline { 2 - 3 } & & Adherenceto medication \\
\cline { 2 - 3 } & & Behavioural activation \\
\hline
\end{tabular}

\subsubsection{Phase I: List of Problems and Working Alliance}

The first three sessions focused on construction of working alliance. In addition, a list of problems reported by the patient was identified. Working together, the therapist and the patient developed a shared ierarchy of goals for the therapeutic intervention. The following goals were identified: (a) to prevent relapses in further psychotic episodes, (b) to improve global functioning, (c) to reduce post-psychotic symptoms of social anxiety and depression.

\subsubsection{Phase I: Case Formulation}

Subsequent sessions (IV-V) were conducted to identify vulnerability, precipitating, maintenance, and protective factors with regard to earlier psychotic episodes. In cooperation with the patient, prodromal signs typical of earlier episodes were identified (eg, reduced need for sleep, high mood, ipercinesia, intense negative emotions). Functional analysis allowed developing case formulation, which identified vulnerability, precipitating, and maintenance factors for psychotic symptoms during earlier episodes.

According to the case formulation, poor coping strategies for negative emotions and poor assertiveness played as vulnerability factors. Dysfunctional beliefs, such as perfectionistic beliefs (eg, "I never commit myself enough to find a job"), were assumed to be an additional vulnerability factor. Life events, such as difficulty finding a job and loss of a relationship with a partner, could play as precipitating factors. Either dysfunctional beliefs or poor assertive skills could play as maintenance factors. Poor assertiveness was hypothesized to be associated to use of substances, which played as a strategy to cope with negative emotions by a negative reinforcement mechanism. According to the case formulation, use of substances can attenuate negative emotions. In conclusion, we hypothesized that the combination of such factors could be responsible for the escalation leading to the earlier psychotic episodes for M..

Case formulation was inspired to the model of Morrison [26], and it was tailored on the specific characteristics of the clinical case.

The case formulation model used during sessions is presented in Figure 1.

\section{Activating event}

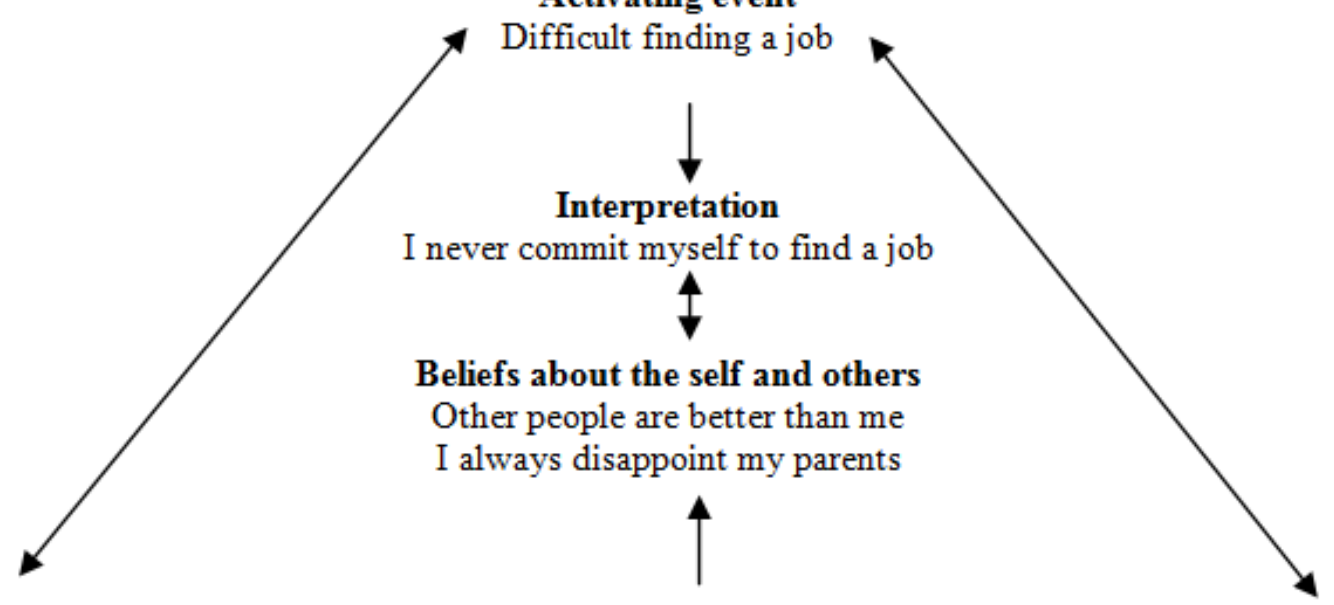

BehavioursVulnerability factorsEmotions

Abuse of cannabis and alcol

Social withdrawalPoor assertiveness and social skillsFrustration

Aggressive behavioursAnger

Figure 1. Case formulation of psychotic episodes of M. (from Morrison, 2001) 


\subsubsection{Phase II: Psichoeducation on Vulnerability} Factors for Psychotic Episodes

During sessions 6-7 psychoeducation was offered in order to increase insight of the patient with regard to prodromal signs of earlier episodes (eg, reduced need for sleep, intense negative emotions etc.). The patient was invited to keep a self-monitoring diary to check daily course of symptoms, such as number of hours spent sleeping, mood states or other typical behaviours. This intervention aimed to increase self-efficacy of the patient to manage potential relapses. Using such case formulation it was possible to show to the patient functional connections between activating events (eg, lack of a job), dysfunctional beliefs (eg, "I never commit myself enough to find a job”), emotions (eg, “anxiety”), and maladaptive coping strategies (eg, "use of substances, social withdrawal”). The patient was assigned with homework and was encouraged to use self-monitoring worksheets to identify further activating events from his daily life (Figure 2).

\section{Automatic though:}

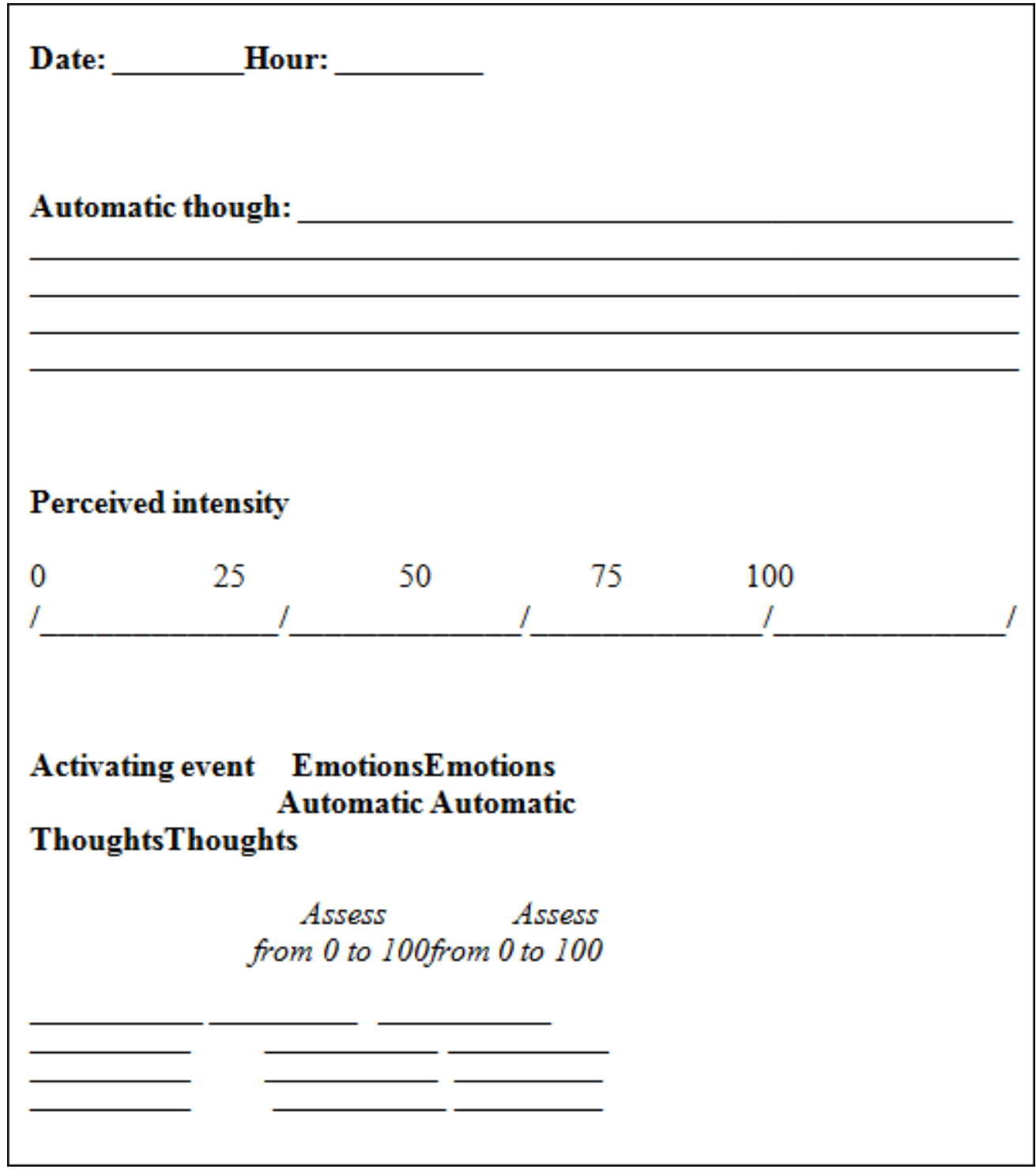

Figure 2. Registration of automatic thoughts

\subsubsection{Phase II: Assertiveness and Social Skills Training}

From sessions 9 to 21 the intervention aimed to increase social skills to cope with negative emotions, since such aspects could be vulnerability factors for further psychotic episodes according to case formulation.

First, psychoeducation was provided with the aim to enhance the awareness of the differences between passive, aggressive, and assertive behaviours. Subsequently, in cooperation with the patient, daily life events were identified for which M. did not have assertive behaviours. Using Socratic dialogue, the therapist suggested to the patient alternative behaviours, which could be adopted in such or similar situations.
In addition, role-playing were conducted by simulating social situations which $M$. had recently faced, such communication difficulties with parents or friends. During role-playing the therapist played as a model and showed to the patient modalities of assertive communication.

In order to optimise the efficacy of psychoeducation, $\mathrm{M}$. was provided with self-help materials regarding verbal and non-verbal aspects of assertive communication. Such materials were assigned as homework and discussed with the therapist during sessions.

Assertiveness training, also, consisted of exercises with worksheets (Figure 3), in which the patient was encouraged to identify activating events, automatic thoughts activated by such events, and passive/aggressive behaviours and emotions 
consequent to those thoughts. In addition, the patient was invited to identify a functional behavioural response.

\begin{tabular}{|c|}
\hline Situation? \\
\hline $\begin{array}{l}\text { Which emotions do you feel in this situation? } \\
\text { [Assess the intensity of each emotion from } 0 \text { to 100] }\end{array}$ \\
\hline Are there thoughts related to this situation? \\
\hline Are there altemative explanations to these thoughts? \\
\hline $\begin{array}{l}\text { Which behaviours have you used to face this situation in response to those } \\
\text { thoughts? } \\
\text { Which are the advantages of such behaviours? Which the disadvantages? }\end{array}$ \\
\hline Whcih are your goals in this situation? \\
\hline Which assertive behaviours could help you to achieve your goals? \\
\hline
\end{tabular}

Figure 3. Self-monitoring of assertive behaviours

Simultaneously, M. was encouraged to choose a by the patient as progressively more anxiety-provoking behaviour, which allowed him to achieve his goals in specific situations. This exercise was proposed as a homework, in which he had to use such strategy in daily situations according to a ierarchy of situations perceived

\section{stimuli.}

\subsubsection{Phase II: Behavioural Activation}

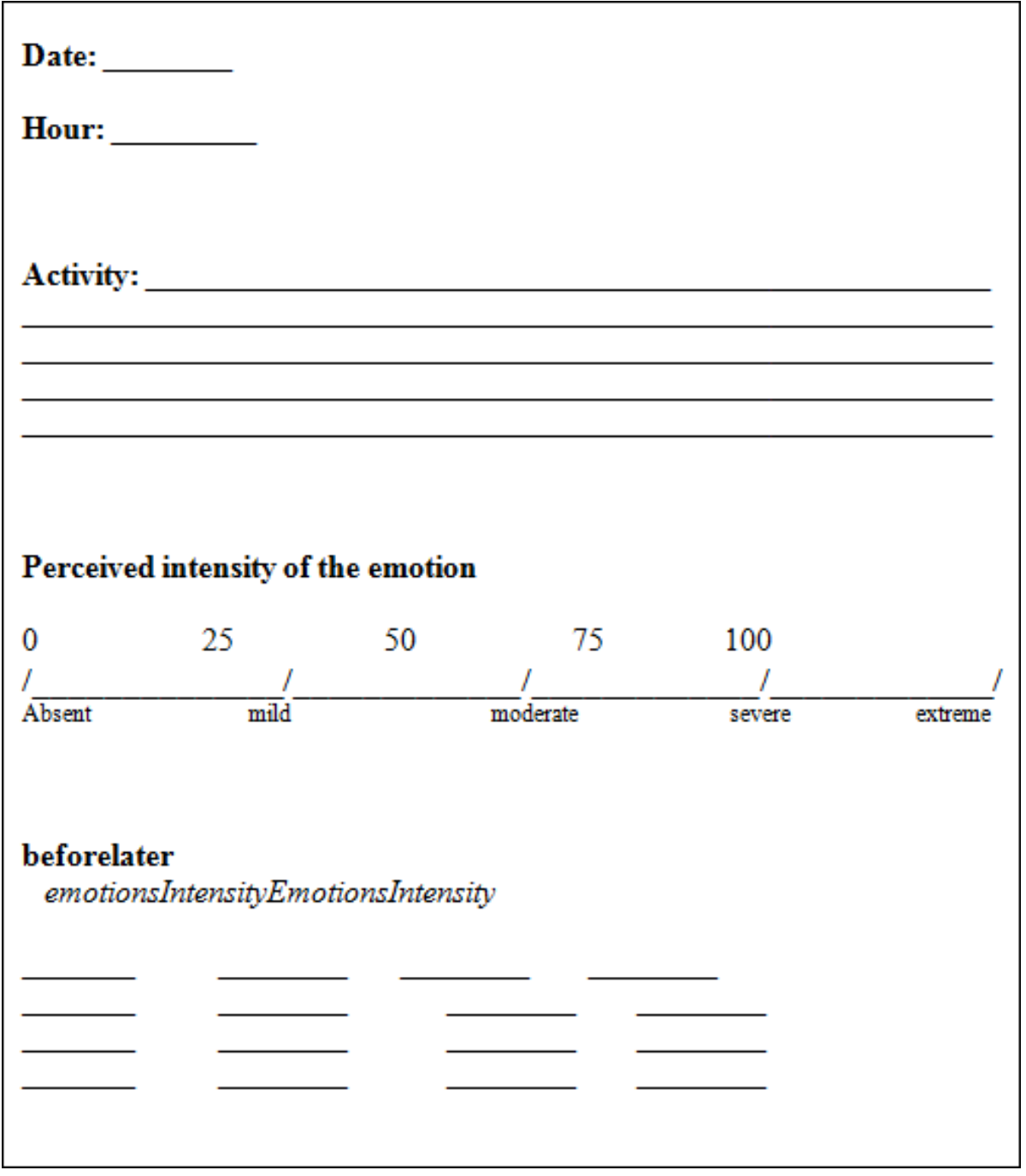

Figure 4. Self-monitoring of behavioural experiments on pleasant activities 
In order to target relapse prevention, homework on scheduling physical and pleasant activities were assigned using a self-monitoring diary (Figure 4). In this diary M. had to indicate and schedule activities he would have engaged during the subsequent day, to report emotions and thoughts he had with regard these activities and to measure the intensity of these emotions. The rationale for these homework was to enhance greater awareness of positive emotions and the intensity associated to them, to enhance self-efficacy in the management of daily living, thus reducing experience of anxiety and depressive symptoms. In order to minimize the negative impact of depressive symptoms, physical activities and meetings with peers were planned. Such actitivies were assigned to M. according to a shared ierarchy, in which the patient was requested to face with activities more and more challenging. Such activities were conceived as behavioural experiments, aimed to challenge the patient's catastrophic beliefs about his capacity to get pleasure from daily activities ("My days always are unemotional”, "I never feel emotions”), stimulating him to distinguish between different emotions and to assess their intensity.

\subsubsection{Phase II: Cognitive Restructuring}

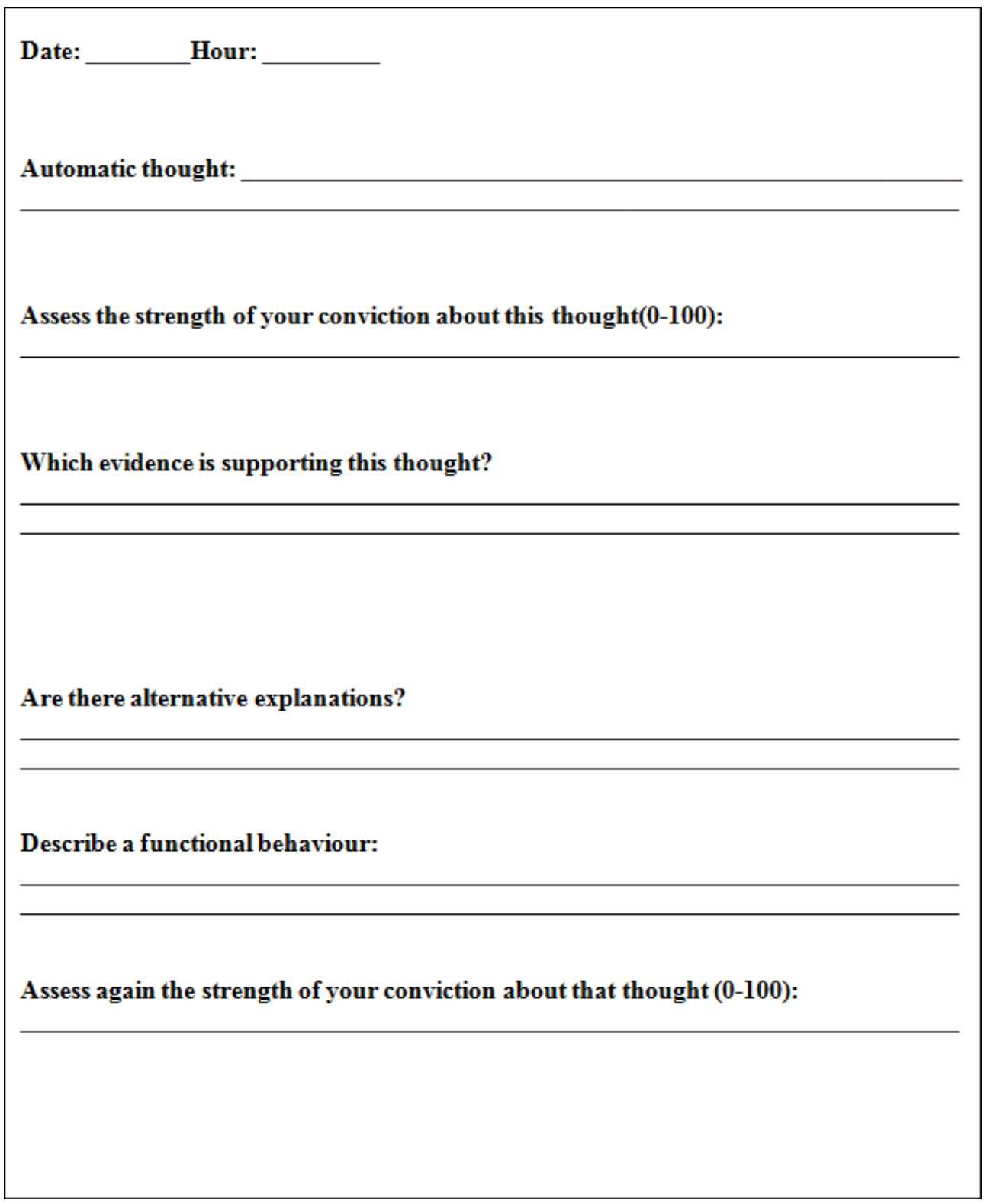

Figure 5. Developing a functional behaviour

Cognitive restructuring (CR) aimed to provide the patient with skills to challenge negative automatic thoughts (i.e. "I am not working enough to find a job”) and to develop alternative explanations in order to help him not to feel entrapped in them, thus reducing the negative impact of depressive symptoms. Such intervention has been conducted during the mid and the final phase of the treatment course. CR was first conducted during sessions, then it was assigned as homework. Using the worksheet provided in Figure 5, M. was encouraged to identify activating events, to distinguish and write emotions and thoughts related to those events and assess their strength. Using Socratic dialogue techniques, the therapist encouraged $\mathrm{M}$. to consider alternative explanations able to disconfirm negative automatic thoughts. Using decision balance sheets, the patient was invited to consider advantages and disadvantages related to believing in those thoughts. Subsequently, M. was helped to find more functional behaviours, which he could have had to face those thoughts. 


\section{Discussion on the Case of $M$.}

Persistence of depressive and social anxiety symptoms represents a relatively common characteristic among patients facing phases subsequent to first psychotic episodes [11,12,13,14]. This aspect may significantly interfere with quality of life of the patients, thereby increasing their vulnerability to relapse in further episodes and to development of a chronic condition [11,12,13,14].

The current study aimed to describe the use of a CBT intervention for the prevention of relapses in psychotic episodes for the clinical case of a young patient who experienced depressive and anxiety symptoms subsequent to first episodes of psychosis. A modular intervention was used, and this approach allowed focusing either on relapse prevention and on psychopathological aspects secondary to psychotic episodes (depression, social anxiety, and poor functioning).

Clinical symptomatology of $\mathrm{M}$. may be considered typical of some patients who experience the onset of psychotic disorders. Such symptomatology often consists of a relatively rapid remission of positive symptoms, enhanced by antipsychotic medications [5,6]. After this remission phase, the patient showed the persistence of depressive and social anxiety symptoms, and during this phase the CBT intervention was started.

During the five months of CBT treatment the patient did not report episodes of psychosis, and this gain was confirmed at a 5-month follow-up. He has shown great adherence with antipsychotics drugs. Interviews conducted at post-treatment and follow-up showed improvement of the patient on global functioning. For example, interviews revealed that the patient began to seek a job, applied to an university post-graduate course, to attend meetings and learning workshops, began a part-time job as a waiter, began to attend a gym, and increased meetings with peers. In addition, the patient seemed to more effectively schedule his daily activities, since at follow-up he told that he continue using behavioural activation worksheets for scheduling activities.

First, the intervention focused on construction of working alliance, with the aim to collaboratively identify a list of problems and set therapeutic goals. The techniques of active listening and reformulation were used to encourage the patient to express his emotions. Working alliance was also supported in order to enhance adherence of the patient with homework assignments. The patient showed strong motivation and readiness to work on the prevention of psychosis episodes, and this aspect played as a protective factor enhancing treatment course.

One of the most important ingredients in first episodes of psychosis treatment, both pharmacological and psychotherapeutic, is represented by working alliance [27]. Among patients with first episodes of psychosis, greater working alliance resulted a significant predictor of a lower perceived severity of positive symptoms by the patient [28], greater global functioning [29], and better adherence with antipsychotic drugs [29]. Given traumatic and severely distressing nature of first episodes of psychosis, the patient may show reactions and symptoms which make him more vulnerable to relapse, such as feelings of shame and guilt or states of severe anxiety related to the possibility of an increase in the dosages of pharmacological drugs or new hospitalizations $[27,28,29]$.
Such reactions may play as factors interfering with helpseeking behaviours, thus increasing feelings of learned helplessness and hopelessness.

During the phase immediately subsequent to the construction of alliance, CBT intervention was based on a case formulation model for psychotic episodes (Figure 1). The model was inspired to the work of Morrison, and it was modified as a function of problems experienced by $\mathrm{M}$. [26]. The model was used to explain to M. vulnerability, precipitating, and maintenance factors for episodes of psychosis. The model was also used to explain the rationale for CBT interventions, and it was discussed during the various stages of treatment with the aim to strengthen the patient's insight about rationale of homework assigned.

During first sessions the patient showed fear of facing further relapses. The use of case formulation aimed to increase perception of self-management with regard to positive symptoms and to reduce feelings of hopelessness. The aim was to normalize dysfunctional beliefs about potential lapses. Potential lapses were presented as a realistic possibility, which could be strongly reduced by completing homework assignments.

In order to normalize dysfunctional beliefs, metaphors were used presenting homework as a self-training, consisting of graded exercises with more and more difficult levels. The use of these metaphors and graded exercises also aimed to empower self-efficacy of the patient with regard to the management of symptoms and to self-distance from hopelessness beliefs.

One of the most critical phases of the treatment was when the patient showed cognitive distortions (eg, "If I feel sad today, I will never be able to get recovered”, "I am not doing enough to get recover”). Following CBT case formulation provided by Morrison [26], it was hypothesized by the therapist that these biased causal attributions could contribute to increase vulnerability of the patient towards further prodromal states.

During mid-treatment the patient showed strongly impairing depressive symptoms, consisting of severely low mood, abulia, apathia, anedonia, feelings of hopelessness and self-blame, and social withdrawal. The intervention developed in phase II focused on this aspect. Functional analysis allowed to understand that such depressive symptoms were activated by dysfunctional beliefs identified as vulnerability factors for psychotic episodes ("I am not working enough to get recovered", "Compared to other people I never am a reactive person”). Such beliefs activated a sort of ruminative escalation, by which the patient tended to self-blame for having made his parents to suffer due his psychotic episodes. Through a negative vicious cycle, such escalation lead to a worsement in depressive symptoms, since the patient progressively withdrawed from social contacts and pleasant activities, dedicating a great part of the day to an uncontrollable rumination. Indeed, social withdrawal tended to confirm dysfunctional beliefs. To address this mechanism, a psychoeducative intervention was used, through which the patient was reminded with regard to the maintenance mechanisms for depressive symptoms. Subsequently, with the aim to reduce depressive symptoms and to modify dysfunctional beliefs associated to them, behavioural activation exercises were introduced. In cooperation with M., a ierarchy of progressively more 
engaging pleasant situations, which were introduced as behavioural experiments to challenge beliefs related to his capacity to get pleasure from activities. Such exercises also aimed to gradually subtract time from rumination which played as a maintenance factor for depression. During interviews at follow-up $\mathrm{M}$. revealed that he felt like more capable to manage negative automatic thoughts and that distress associated to them was progressively decreased.

\subsection{Limitations of the Current Case Report and Implications for Research}

Despite promising gains shown by the patient during interviews at post-treatment and follow-up, it is very important to highlight several strong limitations of our study.

First, our case report lack standardized assessments based on validated measures, which allow to evaluate the effects of the CBT intervention on outcomes. Future studies should use measures of positive symptoms (eg, Psychotic Symptom Rating Scales), post-psychotic depressive symptoms (eg, Calgary Depression Scale), dysfunctional beliefs specifically enhancing psychotic episodes (eg, Cognitive Biases for Psychosis questionnaire), assertiveness skills and social anxiety.

A further limitation concerns the fact that interviews were conducted immediately at the end of treatment and at a 5-month follow-up. Future research should use longer follow-up assessments to examine positive symptoms (or relapse in further episodes), depression, anxiety, and functioning.

In order to investigate the effectiveness of the CBT intervention on depressive and social anxiety symptoms as a relapse prevention approach, future studies should also use a research design based on a randomized controlled trial.

\section{Conflict of Interest}

The authors have no conflict of interest to declare.

\section{References}

[1] Hutton P, Taylor PJ. Cognitive behavioural therapy for psychosis prevention: a systematic review and meta-analysis. Psychol Med 2013; $1-20$.

[2] Schimmelmann BG, Huber CG, Lambert M, et al. Impact of duration of untreated psychosis on pre-treatment, baseline, and outcome characteristics in an epidemiological first-episode psychosis cohort. J Psychiatr Res 2008; 42: 982-990.

[3] Marshall M, Lewis S, Lockwood A, et al. Association between duration of untreated psychosis and outcome in cohorts of firstepisode patients. Arch Gen Psychiatry 2005; 62: 975-983.

[4] Cocchi A, Mapelli V, Meneghelli A, et al. Cost-effectiveness of treating first-episode psychosis: five-year follow-up results from an Italian early intervention programme. Early Intervention in Psychiatry 2011; 5: 203-211.

[5] Kahn RS, Fleischhacker WW, Boter H, et al. Effectiveness of antipsychotic drugs in first-episode schizophrenia and schizophreniform disorder: an open randomized clinical trial. The Lancet 2008; 371: 1085-1097.

[6] National Institute for Health and Clinical Excellence. The NICE guideline on core interventions in the treatment and management of schizophrenia in adults in primary and secondary care. NICE 2009.

[7] Leucht S, Arbte S, Engel RR. How effective are secondgeneration anti-psychotic drugs? A meta-analysis of placebocontrolled trials. Mol Psychiatry 2009; 14: 429-447.

[8] Spencer E, Birchwood M, McGovern D. Management of first episode psychosis. Advances in Psychiatric Treatment 2001; 7: 133-142.

[9] Penn DL, Waldheter EJ, Perkins DO, et al. Psychosocial treatment for first episode psychosis: A research update. Am J Psychiatry 2005; 162: 2220-2232.

[10] Addington J, Gleeson J. Implementing cognitive behavioural therapy for first episode psychosis. Br J Psychiatry 2005; 187(Suppl. 48): 72-76.

[11] Yung AR, Phillips LJ, McGorry PD et al. Prediction of psychosis: a step towards indicated prevention of schizophrenia. $\mathrm{Br} \mathrm{J}$ Psychiatry 1998; 172: 14-20.

[12] Birchwood M, Iqbal Z, Upthegrove R, et al. Psychological pathways to depression in schizophrenia: Studies in acute psychosis, post psychotic depression and auditory hallucinations. Eur Arch Psychiatry Clin Neurosci 2005; 255: 202-212.

[13] Iqbal Z, Birchwood M, Chadwick P, et al. Cognitive approach to depression and suicidal thinking in psychosis: 2. Testing the validity of social rank model. Br J Psychiatry 2000; 177: 522-528.

[14] Romm KL, Melle I, Thoresen C, et al. Severe social anxiety in early psychosis is associated with poor premorbid functioning, depression, and reduced quality of life. Compr Psychiatry 2012; 53: 434-440.

[15] Turner DT, van der Gaag M, Karyotaki E, et al. Psychological interventions for psychosis: A meta-analysis of comparative outcome studies. Am J Psychiatry 2014.

[16] Alvarez-Jimenez, M, Parker AG, Hetrick SE, et al. Preventing the second episode: a systematic review and meta-analysis of psychosocial and pharmacological trials in first-episode psychosis. Schizophr Bull, 2011; 37: 619-630.

[17] Fowler D, Hodgekins J, Painter M, et al. Cognitive behavior therapy for improving social recovery in psychosis: A report from the ISREP MRC Trial Platform Study (Improving Social Recovery in Early Psychosis). Psychol Med 2009; 39: 1627-1636.

[18] Steel C. (Ed.). CBT for Schizophrenia: Evidence-based Interventions and Future Directions. Chichester: John Wiley \& Sons 2013.

[19] Lewis S, Tarrier N, Haddock G et al. Randomised controlled trial of cognitive-behavioural therapy in early schizophrenia: acutephase outcomes. Br J Psychiatry 2002; 181: s91-s97.

[20] Haddock G, Lewis S. Psychological interventions in early psychosis. Schizophr Bull, 2005; 31: 697-704.

[21] Morrison AP. Cognitive behaviour therapy for first episode psychosis: Good for nothing or fit for purpose? Psychosis 2009; 1: 103-112.

[22] Cocchi A, Meneghelli A, Preti A. Programma 2000: celebrating 10 years of activity of an Italian pilot programme on early intervention in psychosis. Aust New Z J Psychiatry 2008; 42: 1003-1012.

[23] Oosthuizen P, Emsley R, Niehaus D, Koen L, Chiliza B. The relationships between depression and remission in first-episode psychosis. World Psychiatry 2006, 5:172-176.

[24] Sönmez N, Hagen R, Andreassen et al. Cognitive Behavior Therapy in First-Episode Psychosis with a Focus on Depression, Anxiety, and Self-Esteem. Cognitive and Behavioral Practice in press.

[25] Gumley AI, Park C. Relapse prevention in early psychosis. In: French P., Reed M, Smith J, Raine M, Shiers D. (Eds.), Promoting recovery in early psychosis: A practice manual. Chichester: Wiley-Blackwell 2010, p. 157-167.

[26] Morrison AP. The interpretation of intrusions in psychosis: an integrative cognitive approach to hallucinations and delusions. Behavioural and Cognitive Psychotherapy 2001; 29: 257-276.

[27] McCabe R, Priebe S. The therapeutic relationship in the treatment of severe mental illness: A review of methods and findings. Int $\mathrm{J}$ Soc Psychiatry 2004; 50: 115-128.

[28] Neale MS, Rosenheck RA. Therapeutic alliance in VA intensive case management. Psychiatr Serv 2005; 46: 719-721.

[29] Davis L, Lysaker P. Therapeutic alliance and improvements in work performance over time in patients with schizophrenia. J Nerv Ment Dis 2007; 195: 4. 